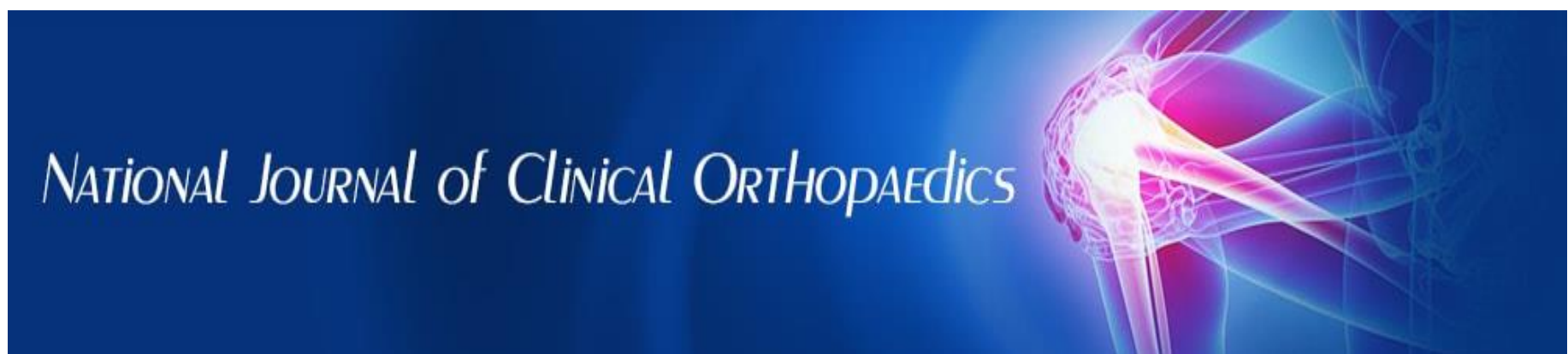

ISSN (P): 2521-3466

ISSN (E): 2521-3474

(C) Clinical Orthopaedics www.orthoresearchjournal.com 2020; 4(2): 01-03

Received: 05-02-2020

Accepted: 09-03-2020

Dr. Tashi G Khonglah Assistant Professor, Department of Orthopaedics \& Trauma,

The North Eastern Indira Gandhi Regional Institute of Health \& Medical Sciences, Mawdiangdiang, Shillong, Meghalaya, India

Dr. Bhaskar Borgohain Professor, Department of Orthopaedics \& Trauma, The North Eastern Indira Gandhi Regional Institute of Health \& Medical Sciences, Mawdiangdiang, Shillong, Meghalaya, India
Corresponding Author: Dr. Tashi G Khonglah Assistant Professor, Department of Orthopaedics \& Trauma, The North Eastern Indira Gandhi Regional Institute of Health \& Medical Sciences, Mawdiangdiang, Shillong, Meghalaya, India

\section{A rare localization of a sessile osteochondroma on the scapula causing shoulder pain in an adolescent girl}

\author{
Dr. Tashi G Khonglah and Dr. Bhaskar Borgohain
}

DOI: https://doi.org/10.33545/orthor.2020.v4.i1a.215

\begin{abstract}
Osteochondromas are common primary bone tumors which are usually found in the metaphyseal region of tubular long bones. The distal femur, proximal tibia and the proximal humerus account for $90 \%$ of the common sites of occurrence. Their occurrence in flat bones such as the pelvis and the scapula are rare and constitute only $3-4 \%$ of all osteochondromas. While the scapula is rarely involved, this is the most common tumor of the scapula. Clinical signs of this lesion include shoulder pain and limited range of motion. This tumor may also present with winging of the scapula. We report a case of a 15-year-old girl presenting with a large sessile osteochondroma of the scapula causing pain on shoulder movements. While osteochondromas of the scapula are rare, it is important to recognise that the most common benign bone tumor of the scapula is an osteochondroma. Due to the unusual location with unspecific shoulder pain, the diagnosis may be hindered. Osteochondromas may have different symptoms depending on their location. Good functional outcomes can be expected following excision of scapular osteochondromas as evidenced by this patient. The prognosis is mostly excellent following excision, but the possibility of recurrence must be kept in mind if the excision is inadequate.
\end{abstract}

Keywords: Benign, bone tumor, exostosis, osteochondroma, scapula, sessile

\section{Introduction}

Osteochondromas are common primary bone tumors which are usually found in the metaphyseal region of tubular long bones ${ }^{[1]}$. The distal femur, proximal tibia and the proximal humerus account for $90 \%$ of the common sites of occurrence ${ }^{[2]}$. Their occurrence in flat bones such as the pelvis and the scapula are rare and constitute only $3-4 \%$ of all osteochondromas ${ }^{[3]}$. While the scapula is rarely involved, this is the most common tumor of the scapula. Clinical signs of this lesion include shoulder pain and limited range of motion. This tumor may also present with winging of the scapula ${ }^{[4]}$.

We report a case of a 15 -year-old girl presenting with a large sessile osteochondroma of the scapula causing pain on shoulder movements.

\section{Case Report}

A 15-year-old girl attending our OPD presented to us with a bulky, swelling over the dorsolateral aspect of her left scapula. The growth was of more than 2 years duration and was progressively increasing in size. On examination, the swelling was located on the dorsolateral border of the left scapula. (Figure 1) It was bony hard and lobulated and moved with her underlying scapula. Movements like abduction and extension of her left shoulder joint were terminally restricted and painful. Although there was no neurovascular deficit, the patient complained of difficulty sleeping in supine. Examination of other common sites of occurrences for such swellings were also found to be normal. Her family history was insignificant. 


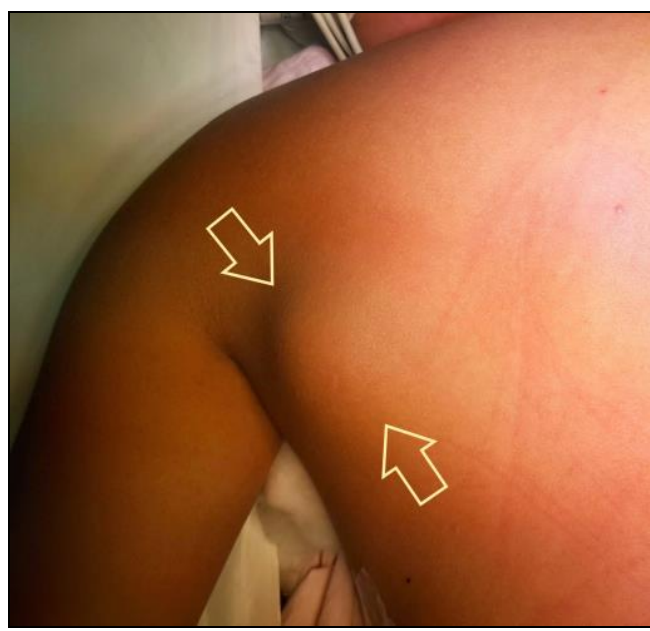

Fig 1: A bony hard swelling arising from the dorsolateral aspect of the left scapula.

A plain radiograph of the affected part showed a large independent, sessile mass lesion arising from the lateral border of the left scapula. (Figure-2) A Computed Tomography (CT) scan revealed a mushroom shaped exostosis measuring 4x6x3 $\mathrm{cm}$ from the infero-lateral border of left scapula. A considerable portion of this bony lesion was found jutting on the ventral aspect of the scapula. (Figure-3\&4) The adjoining soft tissue showed normal density with no lesions seen in either lung.

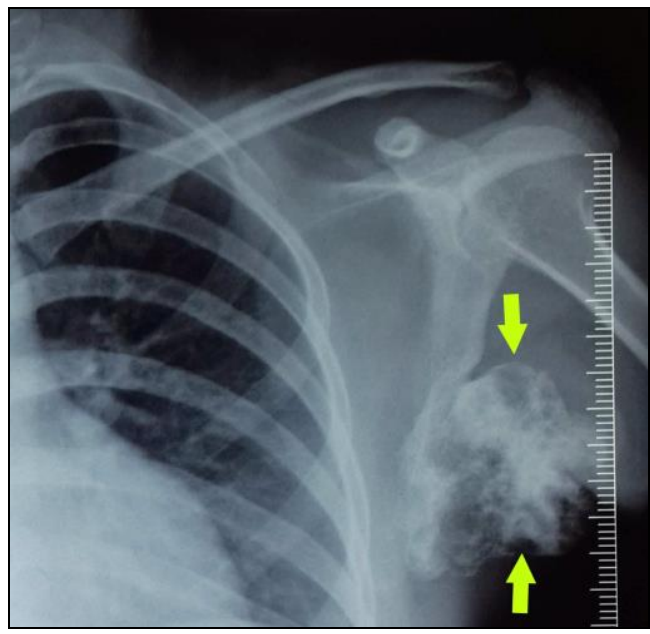

Fig 2: A radiograph showing a large sessile osteochondroma arising from the lateral border of the scapula.

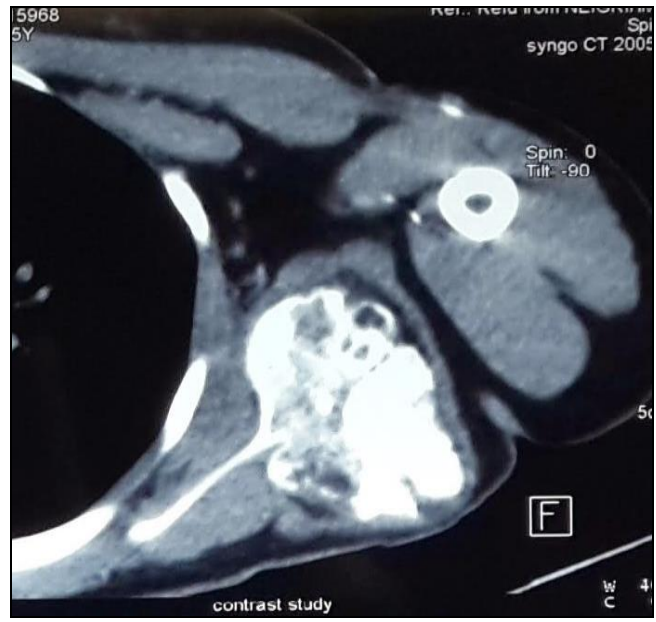

Fig 3: A cross-sectional CT scan showing a large mushroom like growth encroaching the ventral aspect of the scapula.

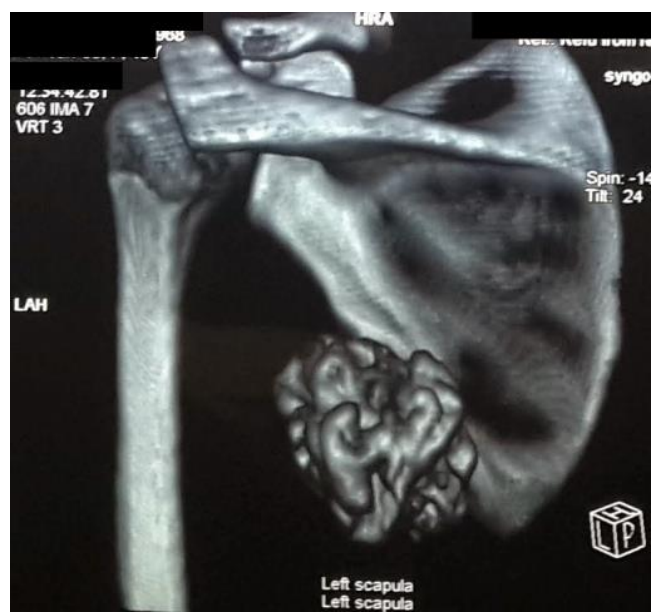

Fig 4: A three-dimensional CT image exhibiting the extent of the tumor on the scapula.

Following this complaint of pain and inability to freely move her left shoulder, the patient was taken up for surgery. In the prone position, under general anesthesia, this bony prominence was resected subperiosteally, using a posterior approach through the interval between the infraspinatus muscle and teres minor. Macroscopically, the tumor appeared knobby without a stalk. (Figure-5\&6) A histopathological examination confirmed the diagnosis of an osteochondroma with no evidence of malignancy.

3 months post-surgery, the patient gained full range of pain free movement on her left shoulder. At 2 years of follow-up we did not find any signs of recurrence.

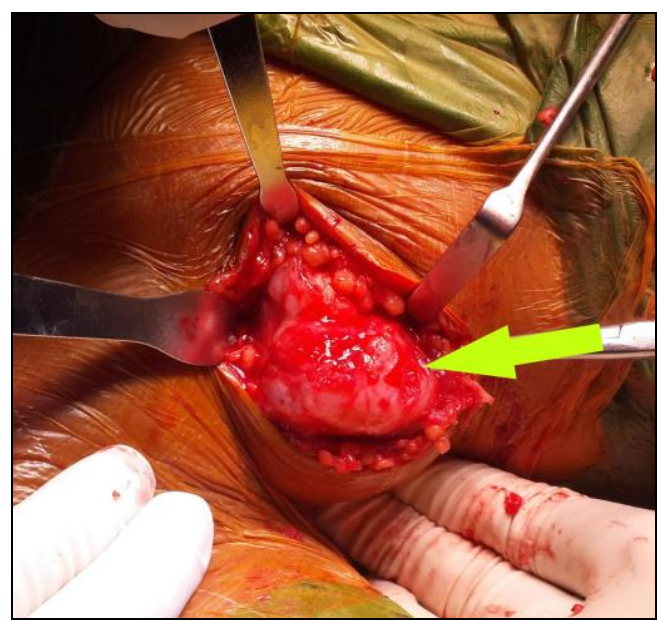

Fig 5: Intraoperative image of the scapular osteochondroma.

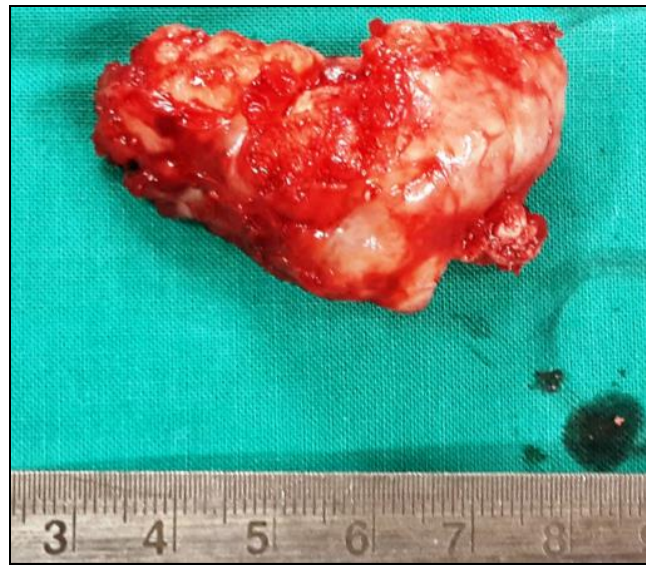

Fig 6: Excised specimen of the bony tumor. 


\section{Discussion}

Osteochondromas account for more than one-third of all benign bone tumors around the world ${ }^{[5]}$. They are usually found in those below 30 years of age, with a male: female ratio of $>1.5: 1$ [6]. They usually present as painless bony swellings found near the meta-epiphyseal regions of long bones like the distal femur, as well as the proximal tibia or humerus in the extremity [5]. Their occurrence in flat bones is relatively rare with scapular lesions accounting for $4 \%$ of all described osteochondroma ${ }^{[7]}$. These osteochondromas account for $14.4 \%$ of all tumours of the scapula making them the most commonly occurring tumor of the scapula ${ }^{[4,7]}$. The ventral surface is involved more commonly with solitary one-sided lesions predominating ${ }^{[8]}$.

Clinical signs of this lesion include shoulder pain, decreased active range of movement, crepitus with movement of the involved shoulder, and "pseudo winging" of the scapula ${ }^{[4]}$. The diagnosis is usually ascertained by the clinical features and by various imagine modalities. This tumor may be confirmed on histopathologic examination ${ }^{[7]}$. Plain radiographs could be a limitation in diagnosing osteochondromas is such locations, hence a CT scan would be useful to display the extent of such

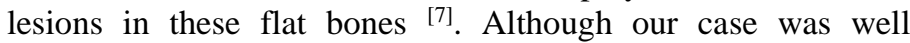
delineated on a plain radiograph, a three-dimensional reconstruction and an axial cut of the lesion on CT scan further assisted in demarcating the lesion and this facilitated planning of the treatment. Magnetic Resonance Imaging is usually saved for cases in which malignant transformation is suspected ${ }^{[9]}$. The treatment for this kind of tumor is open surgery, which does not show any complication when the glenohumeral joint is preserved ${ }^{[8]}$. Following the excision of the tumor, our patient was completely relieved from all symptoms. Sreenivas et al. concluded that preoperative functional restriction in osteochondroma of scapula was mainly due to the location of the tumor especially when present over the ventral or ventromedial aspect scapula ${ }^{[10]}$. Tumor relapse is very rare and usually occurs when unclear resection margins are left ${ }^{[9]}$.

Malignant transformations are extremely rare in children and they are uncommon in adults. The incidence of malignant degeneration in solitary osteochondromas is only $1 \%$ and characterised by sudden increase in size of the lesion accompanied by pain, usually described as severe $[9,11]$. The growing potential of osteochondromas usually ends by the time of closure of the physis. If growth continues into adulthood, it should alarm the surgeon for possible malignancy ${ }^{[9]}$.

\section{Conclusion}

While osteochondromas of the scapula are rare, it is important to recognise that the most common benign bone tumor of the scapula is an osteochondroma. Due to the unusual location with unspecific shoulder pain, the diagnosis may be hindered. Osteochondromas may have different symptoms depending on their location. Good functional outcomes can be expected following excision of scapular osteochondromas as evidenced by this patient. The prognosis is mostly excellent following excision, but the possibility of recurrence must be kept in mind if the excision is inadequate.

\section{References}

1. Vaishya R, Dhakal S, Vaish A. A solitary osteochondroma of the scapula. BMJ Case Rep [Internet]. 2014 Feb 21 [cited 2020 Mar 9], 2014. Available from: https://www.ncbi.nlm.nih.gov/pmc/articles/PMC3931961/

2. Tungdim PH, Singh II, Mukherjee S, Pertin T. Excision of Solitary Osteochondroma on the Ventral Aspect of Left
Scapula Presenting as Pseudowinging in a 4-year-old Boy: A Rare Case Report. J Orthop Case Rep. 2017; 7(1):36-40.

3. Jadhav PU, Banshelkikar SN, Seth BA, Goregaonkar AB. Osteochondromas at Unusual Sites-Case Series with Review of Literature. J Orthop Case Rep. 2016; 6(1):52-4.

4. Galate JF, Blue JM, Gaines RW. Osteochondroma of the scapula. Mo Med. 1995; 92(2):95-7.

5. Tong K, Liu H, Wang X, Zhong Z, Cao S, Zhong C et al. Osteochondroma: Review of 431 patients from one medical institution in South China. J Bone Oncol. 2017; 8:23-9.

6. Chun DI, Cho JH, Choi IH, Yi Y, Kim JY, Kim JH et al. Osteochondroma of ventral scapula associated with chest pain due to rib cage compression: A case report. Medicine (Baltimore). 2018; 97(17):e0510.

7. Kwon OS, Kelly JIV. Delayed presentation of osteochondroma on the ventral surface of the scapula. Int $\mathbf{J}$ Shoulder Surg. 2012; 6(2):61-3.

8. Frost NL, Parada SA, Manoso MW, Arrington E, Benfanti P. Scapular osteochondromas treated with surgical excision. Orthopedics. 2010; 33(11):804.

9. Chillemi C, Franceschini V, Ippolito G, Pasquali R, Diotallevi R, Petrozza V et al. Osteochondroma as a cause of scapular winging in an adolescent: a case report and review of the literature. J Med Case Reports. 2013; 7:220.

10. Sreenivas T, Ravi Kumar N, Nataraj AR. A retrospective analysis of osteochondroma of scapula following excision biopsy. Acta Orthop Belg. 2015; 81(2):303-7.

11. Kumar CY, Shervegar S, Gadi DPR. Solitary sessile osteochondroma of scapula, a rare case report. J Clin Diagn Res JCDR. 2014; 8(3):174-5. 